\title{
Deficits in Decision-Making and reversal learning in college students who participate in Binge drinking
}

\author{
Ji-Yeon Yoo', Myung-Sun Kim ${ }^{\dagger, 1}$
}

\section{ABSTRACT}

Objectives: We investigated deficits in decision-making and reversal learning, and the relationship between them in college students who engaged in binge drinking.

Methods: In total, 30 students involved in binge drinking and 31 non-binge drinking participated. Binge drinking was defined on the basis of the quantity, frequency and speed of alcohol consumption. Decision-making and reversal learning were measured using the lowa gambling task (IGT) and a reversal-learning task, respectively.

Results: The binge drinking group obtained significantly lower total net scores on the IGT versus the non-binge drinking group. Additionally, the binge drinking group obtained significantly lower block net scores in the third and fourth blocks of the IGT, and selected cards from deck B (disadvantageous) more frequently than did the non-binge drinking group. In the reversal-learning task, the binge drinking group had significantly lower accuracy than the non-binge drinking group in the reversal-learning stage but not in the contingency-learning stage. Moreover, associations between performance on the IGT and the reversal-learning task were observed in the binge drinking group but not in the non-binge drinking.

Conclusion: These results indicate that college students participating in BD had impairments in decision-making and reversal learning. Difficulties with reversing previously learned outcomes might lead to repetition of choices that are no longer advantageous.

\section{Keywords}

Binge drinking, Decision-making, lowa gambling task, Reversal learning

\section{Introduction}

Binge drinking (BD) is characterized by a pattern of excessive alcohol consumption followed by a period of abstinence. It has recently gained attention as a major health problem, particularly in college students, because BD is most prevalent among this population [1,2] and because $\mathrm{BD}$ can predict the risk of future alcohol use disorder (AUD) [3]. Patients with AUD and individuals participating in $\mathrm{BD}$ share common abnormalities in brain structure/function and neuropsychological function [4].
One characteristic observed in patients with AUD is that they cannot stop drinking even when they face serious negative outcomes, such as the possibility of losing their job, home, or honor, due to their drinking habits [5]. Because this characteristic is believed to reflect deficits in decision-making [6], and these deficits then cause maladaptive substance use [7], deficits in decision-making in patients with AUD have received attention. Deficits in decision-making are observed not only in patients with chronic AUD [8] but also in patients with fetal alcohol syndrome [9], healthy relatives of patients with

'Sungshin Women's University, Department of Psychology, Seoul, Korea

${ }^{\dagger}$ Author for correspondence: Myung-Sun Kim, Ph. D, Professor, Sungshin Women's University, Department of Psychology, Bomunro 34 dagil 2, Sungbuk, Seoul, Korea; Tel: 82-2-920-7592; Fax: 82-2-920-2040; email: kimms@sungshin.ac.kr 
AUD [10], and individuals with BD [11-13]. Thus, deficits in decision-making are believed to be a trait-marker of AUD.

Decision-making is the process of forming preferences for possible options, selecting and executing actions, and evaluating outcomes [14]. Decision-making is believed to be an important social function in real-life because it requires consideration of future outcomes prior to the selection of actions [13].

The Iowa gambling task (IGT) [15] is widely used to measure decision-making. The IGT is sensitive to risk-reward related decision-making because participants have to learn to sacrifice immediate rewards in favor of long-term benefits. In each IGT trial, participants select a card from 1 of 4 decks; each card selection leads to a gain, which is sometimes coupled with a simultaneous loss. Two decks (A and B) are disadvantageous, and selecting cards from these decks results in an overall net loss, whereas the other two decks (C and D) are advantageous, and selecting cards from these decks results in an overall net gain. The IGT requires that the participants learn the expected value of each deck associated with the response choice while performing the task [16]. Decision-making is measured by the total net score, the block net scores and the total number of cards selected from each deck [17]. Healthy participants choose cards from the advantageous decks more than from the disadvantageous decks as the task progresses [18].

Studies using the IGT to investigate decisionmaking in patients with AUD have shown that patients with AUD chose cards from disadvantageous decks more often than did healthy controls, and that the block net scores of the AUD group did not increase as the test progressed $[8,19]$. Studies that investigated decision-making in college students with BD using the IGT have produced similar results to those with AUD [20,21]. For example, Mullan et al. [20] found that college students with BD performed significantly poorer on the IGT than non-binge drinkers, indicating that binge drinkers had a tendency to make riskier decision. Furthermore, Xiao et al. [12] and Goudriaan et al. [22] observed that the IGT performance of individuals with $\mathrm{BD}$ predicted future drinking problems and heavy drinking, respectively.

Although performance on the IGT has been interpreted in various ways, including myopia for the future [15], it has recently been suggested that impaired performance on the IGT indicates a difficulty in reversal learning [23]. An important aspect of the IGT is that participants have to shift their preference from the decks that are initially rewarding in the earlier trials following subsequent losses [24]. As the immediate gains provided by decks $\mathrm{A}$ and $\mathrm{B}$ are about twice as large as those provided by decks C and D, participants should learn during earlier trials that decks A and B are advantageous. However, as the trials progress, they should recognize that decks $\mathrm{C}$ and $\mathrm{D}$ are more advantageous than are decks $\mathrm{A}$ and $\mathrm{B}$, and should reverse the previously learned contingency $[23,25]$. Studies using the reversallearning task have shown that patients with AUD have deficits in reversal learning [26,27]. Patients with AUD did not show impaired performance in the contingency-learning stage, but showed significantly worse performance after the stimulus contingencies were switched (i.e., the reversal-learning stage) versus healthy controls. These results indicate that patients with AUD have deficits in reversal learning due to a failure to reverse [27] or inhibit [26] previously learned information about the contingencies governing their options. Some studies observed deficits in reversal learning in rats that excessively drank [28], but no study of individuals involved in $\mathrm{BD}$ has been reported.

The ventromedial prefrontal cortex plays an important role in reversal learning because activation in that area increases while healthy controls perform the reversal-learning task [29]. Reduced gray matter volume in the ventromedial prefrontal cortex [30], and associations between total net scores on the IGT and gray matter volume in the ventromedial prefrontal cortex [19] have been observed in patients with AUD. These results indicate that impaired performance on the IGT and a reversal-learning task observed in patients with AUD are related. In BD, significant associations between activation in the orbitofrontal cotex and the severity of drinking problems were observed [13]. However, no study investigating the relationship between performance on the IGT and the reversallearning task in patients with AUD or individuals involved in BD has been reported.

Given this background, we investigated decisionmaking and reversal learning in college students who engaged in BD using the IGT and a reversallearning task. The primary objectives were to investigate whether college students involved in $\mathrm{BD}$ have deficits in decision-making and reversal learning, and if so, whether these deficits were related. In order words, we were interested in 
determining why individuals with $\mathrm{BD}$ continue binge drinking in spite of its associated negative consequences by investigating the relationship between decision-making and reversal learning abilities.

\section{Methods \\ - Participants}

In total, 61 college students were recruited from a pool of 210 students based on their scores on the Korean version of the Alcohol Use Disorder Identification Test (AUDIT-K) [31,32] and the Alcohol Use Questionnaire (AUQ) [33]. BD was defined on the basis of the quantity, frequency and speed of alcohol consumption; drink 5 (male) or 4 (female) units more than once during the previous 2 weeks [34] and drink 3 (male) or 2 (female) units per hour [35].

The World Health Organization recommends a score of $>8$ on the AUDIT as a cut-off [31]; however, others have suggested that the sensitivity and specificity for problem drinking are highest when a score of 12 on the AUDIT is used as cut-off $[32,36]$. Additionally, a score $>26$ on the ADUIT indicates the possibility of alcohol dependence [36]. Therefore, in this study, those who obtained scores of 12-26 on the AUDIT, drank 5 (male) or 4 (female) units more than once during the previous 2 weeks, and drank more than 3 (male) or 2 (female) units per hour (measured by AUQ Item 10), were included in the $\mathrm{BD}$ group $[1,4,35]$. Those who obtained scores $<8$ on the AUDIT, did not drink 5 (male) or 4 (female) units during the last 2 weeks, and drank less than 2 (male) or 1 (female) units per hour, were included in the non-binge drinking (N-BD) group.

The Structured Clinical Interview for DSM-IV Non-Patients (SCID-NP) [37] was administered to ensure that no participant had a history of psychiatric disorder. We also asked some questions to ensure that no participants had medical or neurological disorders. Additionally, the Children of Alcoholics Screening Test (CAST) [38] was administered to identify whether the participants' parents had a history of AUD, and those who obtained a score of $>6$ on the CAST were excluded. The Korean version of the Wechsler Adult Intelligence Scale (K-WAIS) [39] was administered because significant associations between IGT performance and IQ have been reported [40]. It has been reported that young drinkers with depression and anxiety symptoms are at increased risk of AUD during young adulthood [41]. Therefore, depression and anxiety were evaluated using the Self-Rating Depression Scale (SDS) [42] and State-Trait Anxiety Inventory (STAI) [43], respectively.

Based on these criteria, 30 (12 males, 18 females) and 31 (12 males, 19 females) students were included in the $\mathrm{BD}$ and $\mathrm{N}-\mathrm{BD}$ groups, respectively. All participants were instructed to abstain from the use of alcohol for 24 hours prior to the experiment. This study was approved by the Sungshin Women's University Institutional Bioethics Review Board. All participants provided written informed consent after receiving a complete description of the study, and they were paid for their participation at a rate of approximately $\$ 10$ per hour, independently of task performance.

\section{- Alcohol Use Disorder Identification Test (AUDIT)}

The AUDIT [31], a 10-item self-administered questionnaire, was used to measure the quantity and frequency of alcohol consumption, the presence of alcohol dependence, and psychosocial problems related to alcohol consumption. Items $1-8$ and 10 are rated on a 5-point scale $(0-4)$ and Item 9 is rated on a 3 -point scale $(0,2,4)$. Thus, the range of the total scores is $0-40$. Item 10 of the K-AUDIT is rated on a 5-point scale instead of 3-point in original AUDIT, since the Koreans showed significantly higher scores on Item 10 than those in western countries when AUDIT was standardized in Korea [32].

\section{- Alcohol Use Questionnaire (AUQ)}

In this study, items 10,11 , and 12 of the AUQ were used to evaluate the speed of drinking (average drinks per hour), number of times being drunk in the previous 6 months, and percentage of times getting drunk when drinking, respectively [43]. Additionally, the binge score was calculated as $[4 \mathrm{x}($ Item 10) + Item $11+0.2$ $\mathrm{x}$ (Item 12)] in order to estimate the severity of BD [44].

\section{- lowa gambling task (IGT)}

A computerized IGT [17] was administered to measure decision-making. In each trial, 4 decks of cards were presented on a monitor, and the participants selected a card from any of the 4 decks; they were then informed how much was won or lost by choosing that card. When a card was selected, the gain or loss was presented on the monitor. Gains occurred whenever cards were selected, but losses occurred according to 
certain ratios. The decks differed in terms of the magnitudes and frequencies of gains and losses.

Participants were instructed to gain as much as possible by selecting cards resulting in maximum gains and minimum losses, but they were not told which decks were advantageous or disadvantageous. In total, 120 trials, including 20 practice trials, were administered. Decisionmaking ability was measured by total net and block net scores. The total net scores were calculated as the total number of cards selected from advantageous decks minus the total number of cards selected from disadvantageous decks $([\mathrm{C}+\mathrm{D}]-[\mathrm{A}+\mathrm{B}])$. Additionally, the 100 trials were divided into 5 blocks, and the block net scores were calculated in the same way as the total net scores.

\section{- Reversal-learning Task}

To evaluate contingency and reversal learning, the reversal-learning task of Robinson et al. [45] was modified. In each trial, 2 cards depicting a human face and a landscape were presented in the center of a computer monitor, and 1 of 2 cards has black frame; these were randomly presented. Selecting one card resulted in a gain, whereas selecting the other resulted in a loss. Participants were instructed to guess whether the card with the black frame was advantageous or disadvantageous, and if the card with the black frame was advantageous, a green smiling face was presented, whereas if the card with black frame was disadvantageous, a red crying face was presented. Additionally, participants were instructed that advantageous or disadvantageous cards could be changed randomly.

Stimuli were presented using E-Prime (Psychological Software Tools Inc., Sharpsburg, PA, USA). The crosshair was displayed for 1,000 $\mathrm{ms}$, and 2 cards were presented for $1,500 \mathrm{~ms}$. after the participant's response, the green smiling face or the red crying face was presented for 500 ms. when a participant did not respond, a "Too late" massage was presented for $500 \mathrm{~ms}$. The procedure for stimulus presentation is described in Figure 1.

In total, 480 trials were presented in 4 blocks, and each block consisted of contingency-learning and reversal- learning stages. After 6 correct responses were made in a row, the contingency was switched, and each block ended after 8 contingency switches had occurred.

Contingency learning was measured by the average number of trials needed to contingency switch [27] and the accuracy prior to the contingency switch [46]. Reversal learning was evaluated by the number of contingency switches [46] and accuracy in the reversal-learning stage [23]. Trials participants did not respond were excluded from the analysis. Prior to the reversal-learning task, a block of 15 trials was administered to ensure that the instructions were understood. The administration of the IGT and the reversal-learning task was counterbalanced across the participants.

\section{- Statistical analysis}

The demographic characteristics of the BD and $\mathrm{N}-\mathrm{BD}$ groups were compared using independent $t$-tests. Total net scores on the IGT were analyzed with a univariate analysis of variance (ANOVA), and block net scores were analyzed by a mixeddesign ANOVA, with block as a within-subject factor and group as a between-subject factor. Deck selection was also analyzed with a mixed-design ANOVA, treating the deck as a within-subject factor and group as a between-subject factor. For the reversal-learning task, the behavioral data from the contingency-learning stage (accuracy and number of trials needed to contingency switch) and reversal-learning stage (accuracy and number of contingency switches) were analyzed using independent $t$-tests. Associations between performances on the IGT and reversal-learning task and between the severity of BD and performance on the IGT/reversal-learning task were analyzed using Pearson product correlations. An alpha level of 0.05 was applied for statistical analysis. All statistical analyses were carried out using SPSS software version 20 .

\section{Results}

\section{- Demographic characteristics}

The BD and N-BD groups did not differ in terms of age, educational level, IQ, SDS, state anxiety of the STAI, trait anxiety of the STAI, or CAST. However, the two groups differed in terms of the AUDIT score, speed of drinking, number of times being drunk in the previous 6 months, percentage of times getting drunk when drinking, and binge score on the AUQ. The BD group showed significantly higher scores on these measures than the N-BD group. The demographic characteristics of the two groups are presented in Table 1.

\section{- The IGT}

The BD group had significantly lower total net scores than the N-BD group $(F(1,59)=5.45$,

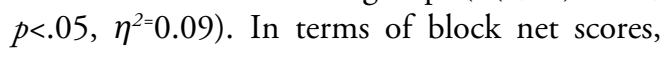




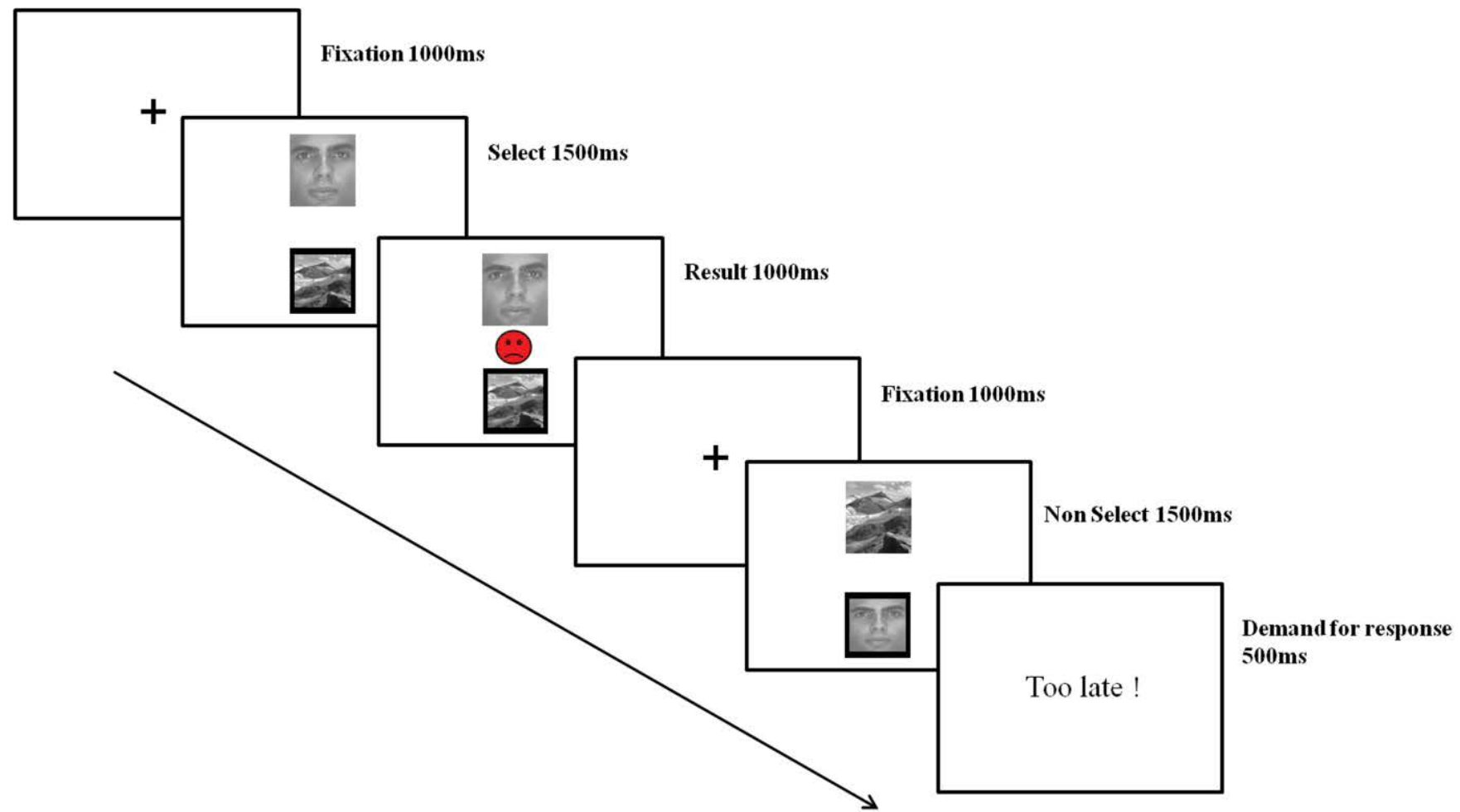

Figure 1: The procedure of stimulus presentation in the reversal-learning task.

main effects of group $(F(1,59)=5.45, p<.05$, $\eta^{2=0.09)}$ and block $(F(4,236)=11.63, p<.001$, $\eta^{2=0.17)}$ were observed, with the $\mathrm{BD}$ group receiving significantly lower block net scores than the N-BD group; the highest and lowest block net scores were observed in the fourth and first blocks, respectively. The block $\mathrm{x}$ group interaction was also significant $(F(4,156)=3.38$,

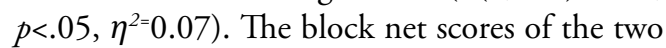
groups for each block were analyzed further using independent $t$-tests. The block net scores of the $\mathrm{BD}$ and $\mathrm{N}-\mathrm{BD}$ groups did not differ in the first $(t(59)=-1.18$, ns, $d=-0.30)$, second $(t(59)=-1.90, n s, d=-0.49)$, or fifth $(t(59)=-0.74$, $n s, d=-0.19)$ blocks. However, individuals with BD obtained significantly lower block net scores in the third $(t(59)=-2.39, p<.05, d=-0.61)$ and fourth $(t(59)=-1.97, \quad p<.05, d=-0.56)$ blocks. Figure 2 presents the mean total net and block net scores of the BD and N-BD groups.

In terms of deck selection, a significant group $\mathrm{x}$ deck interaction was observed $(F(3,177)=2.64$, $\left.p<.05, \eta^{2}=0.04\right)$. Specifically, the two groups differed in the selection of $\operatorname{deck} B$, with the $\mathrm{BD}$ group selecting cards from deck $\mathrm{B}$ more frequently than the N-BD group. The mean number of cards selected from each of the four decks is presented in Table 2.

\section{- Reversal-learning task}

There was no significant difference between the $\mathrm{BD}$ and N-BD groups in the number of trials needed to contingency switch or accuracy in the contingency-learning stage. However, the two groups differed in terms of accuracy in the reversal-learning stage, where the $\mathrm{BD}$ group showed significantly lower accuracy than the N-BD group. Performances on the reversallearning task are presented in Table 3.

\section{- Correlations between performances on the IGT and reversal-learning task}

For the $\mathrm{BD}$ group, significant associations between the number of trials needed for contingency switching in the contingencylearning stage and fifth block score of the IGT $(r=-.47, p<.01)$ and between the number of contingency switches in the reversallearning stage and fifth block score of the IGT $(r=.46, p<.05)$ were observed; more trials in the contingency-learning stage and fewer contingency switches in the reversalleaning stage, and lower fifth block scores on the IGT in the BD group. However, no significant association between performance on the reversal-learning task and the IGT was observed in the N-BD group. 


\begin{tabular}{|c|c|c|c|c|}
\hline & $\begin{array}{l}\text { Binge group } \\
\text { gggrggrgrggrgroudrinking group }\end{array}$ & Non-binge group & \multirow{3}{*}{$t$} & Effect size sizes \\
\hline & $(n=30)$ & $(n=31)$ & & \multirow[t]{2}{*}{ (d) } \\
\hline & Mean (SD) & Mean (SD) & & \\
\hline Age(year) & $21.77(2.64)$ & $21.65(2.30)$ & 0.19 & 0.05 \\
\hline Education(year) & $14.63(1.47)$ & $15.13(1.28)$ & -1.40 & -0.36 \\
\hline IQ & $113.83(8.14)$ & $114.71(10.55)$ & -0.36 & -0.09 \\
\hline SDS & $39.57(5.65)$ & $38.90(8.00)$ & 0.37 & 0.10 \\
\hline STAI state & $39.70(8.78)$ & $39.52(10.52)$ & 0.07 & 0.02 \\
\hline STAI trait & $42.10(9.72)$ & $39.45(10.43)$ & 1.03 & 0.26 \\
\hline AUDIT & $16.30(3.08)$ & $1.97(1.68)$ & $22.48^{* * * *}$ & 5.78 \\
\hline Speed of drinking(drinks/hour) & $4.13(1.11)$ & $0.84(0.52)$ & $14.80^{* * *}$ & 3.80 \\
\hline Times drunk in the last 6 months & 4.77 (4.59) & $0.13(0.43)$ & $5.51^{* * * *}$ & 1.42 \\
\hline $\begin{array}{l}\text { Percentage of times became drunk when } \\
\text { drinking (\%)drin when drinking(\%) }\end{array}$ & $41.53(32.50)$ & $13.13(25.39)$ & $3.80^{* * *}$ & 0.97 \\
\hline AUQ binge drinking score & $29.69(10.66)$ & $6.24(5.57)$ & $10.72^{* * * *}$ & 2.76 \\
\hline CAST & $1.70(1.77)$ & $1.00(1.44)$ & 1.70 & 0.43 \\
\hline
\end{tabular}

\section{- Correlation between BD and performance on the IGT/reversal-learning task}

There was no significant association between the severity of BD, as measured by the AUDIT/ AUQ, and performance on the IGT/reversallearning task in the $\mathrm{BD}$ group.

\section{Discussion}

We investigated whether college students involved in $\mathrm{BD}$ had deficits in decision-making and reversal learning, and if so, whether these deficits were related, using the IGT and a reversallearning task. The BD group had significantly lower total net scores than the N-BD group; these results are consistent with previous results [20-22]. Additionally, the BD group showed significantly lower block scores in the third and fourth blocks than the N-BD group.

Successful performance on the IGT requires that participants learn the contingencies of gains and losses, and learning these contingencies requires an extended period of trials because the magnitudes and frequencies of the gains and losses vary across decks [47]. Bechara et al. [48] reported that most healthy controls switched from one deck to another to learn the contingencies via trial and error at the outset; however, after -50 trials (the third block of the IGT), they recognized that decks A and B were disadvantageous and their preferences became biased towards decks with higher net gains (decks $\mathrm{C}$ and D). Bø et al. [49] investigated whether severity of $\mathrm{BD}$ could predict specific cognitive impairments in college students using CANTAB Stop Signal Task, and found that higher binge score derived from the AUQ was associated with less adjustment following failures, i.e., difficulty in adapting to negative consequences. Thus, the present results showing individuals with $\mathrm{BD}$ obtaining lower total net scores and lower block net scores in the third and fourth blocks indicate that these individuals have deficits in decisionmaking and switching their preferences in spite of great losses or negative consequences they experienced.

Additionally, the BD group selected cards from deck B more frequently than the N-BD group. Decks $\mathrm{A}$ and $\mathrm{B}$ are disadvantageous because these decks deliver immediate large gains but ultimately result in an overall net loss, whereas decks $\mathrm{C}$ and $\mathrm{D}$ are advantageous because these decks deliver immediate small gains but results in an overall net gain. Therefore, it was assumed that participants gradually shift their preferences from decks A and B to decks C and B [15]. However, a number of studies reported that even healthy participants preferred deck B to other three decks $[24,50]$. Lin et al. [51] suggested that this "prominent deck B" phenomenon implies that choice behavior on the IGT is dominated by the high-frequency gain rather than large loss of deck B. Johnson et al. [52] administered 
a modified IGT in which each card selection resulted in a loss, which was sometimes coupled with a simultaneous gain occurring in a certain ratio, and found that $\mathrm{BD}$ and $\mathrm{N}-\mathrm{BD}$ groups did not differ in performance with the modified IGT. They suggested that impaired performance on the original IGT observed in individuals with $\mathrm{BD}$ indicated that these individuals were more sensitive to the gains than losses. Additionally, Gullo et al. [53] found that reward sensitivity predicted the severity of drinking problems, and that reward sensitivity was an important risk factor for drinking problems. Further, Bø et al. [54] found that binge score derived from AUQ was associated with performances of the first 40 trials on the IGT in college students, and they suggested that severity of binge drinking is associated with hypersensitivity to reward and disregard of negative consequences. Taken together, the present result indicates that individuals with BD were more sensitive to frequent gains than losses.

Contingency learning and reversal learning play an important role in decision-making. Decisionmaking requires learning the contingencies of gains and losses, and reversing previously learned contingencies, according to environmental changes [55]. The present results for the reversal-learning task showed that the $\mathrm{BD}$ and $\mathrm{N}$-BD groups did not differ in performance in the contingency-learning stage, but in the reversal- learning stage, the $\mathrm{BD}$ group showed significantly lower accuracy than the N-BD group. These results are consistent with previous studies of patients with AUD [26,27], indicating that college students with $\mathrm{BD}$ have difficulties in reversal learning like those with AUD. The orbitofrontal and ventromedial prefrontal cortices are involved in reversal learning [56]. These brain areas are involved in goal-oriented behavior and modifying behavior according to environmental changes [57]. Addictive substances, like alcohol, cause changes in the activations in these areas and deficits in reversal learning [58]. For example, Xiao et al. [13] observed that reduced activation in the orbitofrontal prefrontal cortex was related to drinking problems in the $\mathrm{BD}$ group. Taken together, individuals with $\mathrm{BD}$ seem to have dysfunctional orbitofrontal and ventromedial prefrontal cortices, which could cause deficits in reversal learning.

Furthermore, associations between performances on the IGT and the reversal-learning task were observed in the $\mathrm{BD}$ group. These results indicate that the deficits in decision-making
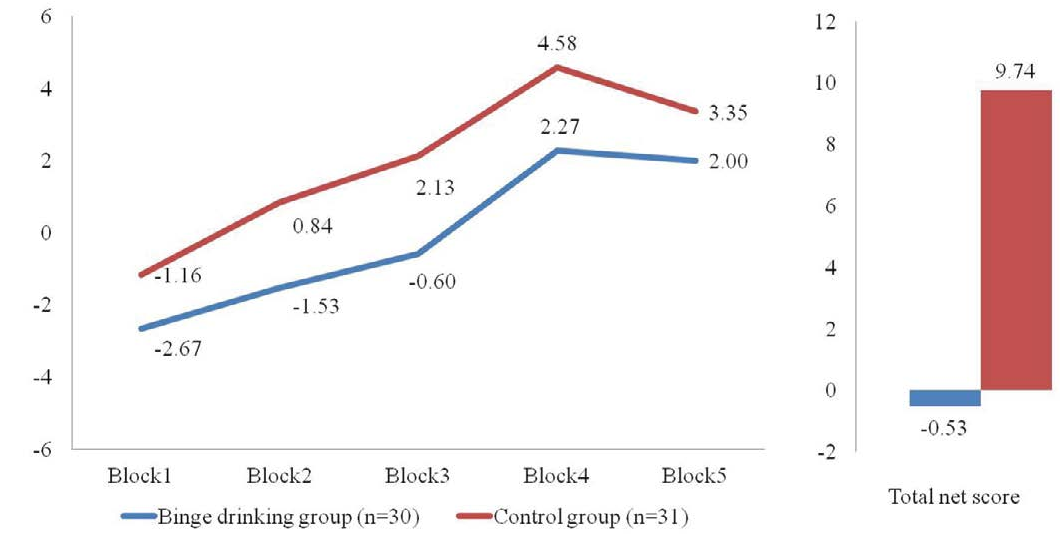

Figure 2: Block net scores (left) and total net score (right) of the IGT in binge and non-binge drinking groups.

\begin{tabular}{|c|c|c|c|c|}
\hline & Binge group & Non-binge group & \multirow{3}{*}{$t$} & Effect size \\
\hline \multirow[t]{2}{*}{ IGT } & $(n=30)$ & $(n=31)$ & & (d) \\
\hline & Mean (SD) & Mean (SD) & & \\
\hline Card A & $19.70(5.17)$ & $19.94(6.56)$ & -0.16 & -0.04 \\
\hline Card B & $30.23(6.76)$ & $25.19(9.11)$ & $2.45^{*}$ & 0.63 \\
\hline Card C & $24.13(7.93)$ & 28.35 (10.70) & -1.75 & -0.45 \\
\hline Card D & $25.93(7.53)$ & $26.52(8.38)$ & -0.29 & -0.07 \\
\hline
\end{tabular}

\section{Table 3: Performance of reversal-learning task in binge and non-binge} drinking groups.

\begin{tabular}{|c|c|c|c|c|}
\hline & Binge group & Non-binge group & \multirow{3}{*}{$t$} & Effect size \\
\hline & \multirow{2}{*}{$\begin{array}{l}(n=30) \\
\text { Mean (SD) }\end{array}$} & $(n=31)$ & & \multirow[t]{2}{*}{ (d) } \\
\hline & & Mean (SD) & & \\
\hline \multicolumn{5}{|l|}{ Contingency learning stage } \\
\hline Average number of trials & $34.30(25.71)$ & $32.77(30.12)$ & 0.21 & 0.05 \\
\hline Accuracy (\%) & $64.87(15.12)$ & $66.61(17.63)$ & -0.42 & -0.11 \\
\hline \multicolumn{5}{|l|}{ Reversal learning } \\
\hline Number of reversals & $15.90(11.43)$ & 20.35 (11.48) & -1.52 & -0.39 \\
\hline Accuracy (\%) & $66.10(16.50)$ & 75.06 (16.79) & $-2.10^{*}$ & -0.54 \\
\hline \multicolumn{3}{|l|}{${ }^{*} p<.05$} & & \\
\hline
\end{tabular}

observed in individuals with $\mathrm{BD}$ are related to the deficits in reversal learning. In other words, these results indicate that individuals engaged in $\mathrm{BD}$ have deficits in reversing previously learned information about the contingencies governing option, as also found in previous studies [25], which lead to deficits in decision-making [23].

In the present study, associations between the severity of $\mathrm{BD}$ and performance on the IGT were not observed in the $\mathrm{BD}$ group. These 
results differ from those of previous studies. For example, Xiao et al. [12] reported that impaired performance on the IGT predicted the quantity and frequency of drinking 1 year later in a BD group. In a longitudinal study, Goudriaan et al. [11] found associations between heavy drinking and impaired performance on the IGT in 18-19-year-old but not 20-21-year-old participants. These results indicate that excessive consumption of alcohol affects decision-making more in adolescents than in young adults. The prefrontal areas involved in decision-making develop most during late adolescence [59], and alcohol has detrimental effects on the developing brain [60]. It is possible that because the college students who engaged in $\mathrm{BD}$ and participated in the present study were adults, with a mean age of 21.77 years, we did not find any associations between $\mathrm{BD}$ and performance on the IGT.

No relationship between the severity of BD and performance on the reversal-learning task in the $\mathrm{BD}$ groups was observed. Gullo et al. [53] administered the reversal-learning task to college students who engaged in hazardous drinking, and found associations between impulsivity but not the severity of hazardous drinking and performance on the reversal-learning task. In addition, Adan et al. [61] observed that male college students with $\mathrm{BD}$ showed higher impulsivity than controls. In this study impulsivity was not assessed.

Our study has several limitations that should be addressed in the future. First, the small number of participants limits the generalizability of these findings. Second, because alcohol produces more detrimental effects in the female than in the male brain [62], separately investigating performance on the IGT and reversal-learning task in female and male students involved in BD is needed. Third, previous studies have suggested that deficits in decision-making cause drinking problems $[7,22$,$] , and that excessive drinking causes$ changes in cortical activation, leading to deficits in decision-making [58]. Therefore, longitudinal studies using neuroimaging techniques are important to understand the neural mechanisms underlying the deficits in decision-making observed in individuals with AUD and involved in BD. Finally, college students are more likely to use other substances, including cigarettes and marijuana [63], controlling these substances is needed in future studies.

In conclusion, students participating in $\mathrm{BD}$ showed impairments in decision-making and reversal learning. Difficulties with reversing previously learned outcomes might lead to repetition of choices that are no longer advantageous. The present results might give some explanations why binge drinking continues in spite of its associated negative consequences.

\section{Acknowledgements \\ This work was supported by the Ministry of Education of the Republic of Korea and the National Research Foundation of Korea (NRF- 2015S1A5A2A03047656).}

\section{Competing and conflicting interests}

There is no conflict of interest.

\section{References}

1. Parada M, Corral M, Mota N, et al. Executive functioning and alcohol binge drinking in university students. Addict. Behav 37(2), 167-172 (2012)

2. Squeglia LM, Schweinsburg AD, Pulido $C$, et al. Adolescent binge drinking linked to abnormal spatial working memory brain activation: differential gender effects. Alcohol. Clin. Exp. Res 35(10), 1831-1841 (2011).

3. Bonomo YA, Glenn B, Carolyn C. Teenage drinking and the onset of alcohol dependence: A cohort study over seven years. Addiction 99(12), 1520-1528 (2004).

4. Mota N, Parada M, Crego A, et al. Binge drinking trajectory and neuropsychological functioning among university students: $\mathrm{A}$ longitudinal study. Drug. Alcohol. Depend
33(1), 108-114 (2013).

5. American Psychiatric Association. Diagnostic and Statistical Manual of Mental Disorders (DSM-5). Washington DC: American Psychiatric Association (2013).

6. Bechara A. Risky business: emotion, decision-making, and addiction. J. Gambl. Stud 19(1), 23-51 (2003).

7. Bechara A. Decision making, impulse control and loss of willpower to resist drugs: A neurocognitive perspective. Nature. Neurosci 8(11), 1458-1463 (2005).

8. Dom G, De Wilde B, Hulstijn W, et al. Decision-making deficits in alcoholdependent patients with and without comorbid personality disorder. Alcohol. Clin. Exp. Res 30(10), 1670-1677 (2006).

9. Kully-Martens K, Treit S, Pei J, et al. Affective decision-making on the lowa Gambling
Task in children and adolescents with fetal alcohol spectrum disorders. J. Int. Neuropsychol. Soc 19(02), 137-144 (2013).

10. Dolan SL, Bechara A, Nathan PE. Executive dysfunction as a risk marker for substance abuse: the role of impulsive personality traits. Behav. Sci. Law 26(6), 799-822 (2008).

11. Goudriaan AE, Grekin ER, Sher KJ. Decision making and binge drinking: A longitudinal study. Alcohol. Clin. Exp. Res 31(6), 928-938 (2007).

12. Xiao L, Bechara A, Grenard LJ, et al. Affective decision making predictive of Chinese adolescent drinking behaviors. J. Int. Neuropsychol. Soc 15, 547-557 (2009).

13. Xiao L, Bechara A, Gong Q, et al. Abnormal affective decision making revealed in adolescent binge drinkers using a functional magnetic resonance imaging study. Psychol. Addict. Behav 27(2), 1-12 
(2012).

14. Ernst M, Paulus MP. Neurobiology of decision making: A selective review from a neurocognitive and clinical perspective. Biol. Psychiatry 58(8), 597-604 (2005).

15. Bechara A, Damasio AR, Damasio H, et al. Insensitivity to future consequences following damage to human prefrontal cortex. Cognition 50(1), 7-15 (1994).

16. Clark L, Robbins TW. Devision-making. In: Wood SJ, Allen NB, Pantelia C. (Eds). The neuropsychology of mental illness. Cambridge: Cambridge University Press, 138156 (2009).

17. Bechara A. lowa gambling task professional manual. Lutz FL: Psychological Assessment Resources (2007)

18. Struglia F, Stratta P, Gianfelice D, et al. Decision-making impairment in schizophrenia: relationships with positive symptomatology. Neurosci. Lett 502(2), 80-83 (2011).

19. Le Berre AP, Rauchs $G$, La Joie R, et al. Impaired decision-making and brain shrinkage in alcoholism. Eur. Psychiatry 29(1), 125-133 (2014)

20. Mullan B, Wong C, Allom V, et al. The role of executive function in bridging the intentionbehavior gap for binge drinking in university student. Addict. Behav 36(10), 1023-1026 (2011).

21. Moreno M, Estevez AF, Zaldivar F, et al. Impulsivity differences in recreational cannabis users and binge drinkers in a university population. Drug. Alcohol. Depend 124(3), 355-362 (2012).

22. Goudriaan AE, Grekin ER, Sher KJ. Decision making and response inhibition as predictors of heavy alcohol use: a prospective study. Alcohol. Clin. Exp. Res 35(6), 1050-1057 (2011).

23. Fellows LK, Farah MJ. Different underlying impairments in decision-making following ventromedial and dorsolateral frontal lobe damage in humans. Cereb. Cortex 15(1), 58-63 (2005).

24. Dunn BD, Dalgleish T, Lawrence AD. The somatic marker hypothesis: A critical evaluation. Neurosci. Biobehav. Rev 30(2), 239271 (2006)

25. Maia TV, McClelland JL. A reexamination of the evidence for the somatic marker hypothesis: what participants really know in the lowa Gambling Task? Proc. Natl. Acad. Sci. USA 101(1), 16075-16080 (2004).

26. Fortier CB, LaFleche G, Disterhoft JF, et al. Delay discrimination and reversal eyeblink classical conditioning in abstinent chronic alcoholics. Neuropsychology 22(2), 196-208 (2008).

27. Vanes LD, Holst RJ, Jansen JM, et al. Contingency learning in alcohol dependence and pathological gambling: learning and unlearning reward contingencies. Alcohol. Clin. Exp. Res 38(6), 1602-1610 (2014).

28. Coleman LG, He J, Lee J, et al. Adolescent binge drinking alters adult brain neurotransmitter gene expression, behavior, brain regional volumes, and neurochemistry in mice. Alcohol. Clin. Exp. Res 35(4), 671-688 (2011).

29. Cools R, Clark L, Own AM, et al. Defining the neural mechanisms of probabilistic reversal learning using event-related functional magnetic resonance imaging. J. Neurosci 22(11), 4563-4567 (2002).

30. Cardenas VA, Durazzo TC, Gazdzinski S, et al. Brain morphology at entry into treatment for alcohol dependence is related to relapse propensity. Biol. Psychiatry 70, 561-567 (2011).

31. Babor TF, Higgins-Biddle JC, Saunders JB, et al. AUDIT: The Alcohol Use Disorders Identification Test: Guidelines for use in primary care, second edition. Geneva: World Health Organization (2001).

32. Lee BO, Lee CH, Lee PG, et al. Development of Korean version of Alcohol Use Disorders Identification Test (AUDIT-K): Its reliability and validity. J. Kor. Acad. Addict. Psychiatry 4(2), 83-92 (2000)

33. Mehrabian A, Russell JA. A questionnaire measure of habitual alcohol use. Psychol. Reports 43(3), 803-806 (1978).

34. Wechsler $\mathrm{H}$, Nelson TF. Binge drinking and the American college student: What's five drinks? Psychol. Addict. Behav 15(4), 287-291 (2001).

35. National Institute on Alcohol Abuse and Alcoholism (NIAAA). NIAAA council approves definition of binge drinking. NIAAA Newsletter, 3 (2004).

36. Kim JS, Oh MK, Park BK, et al. Screening criteria of alcoholism by alcohol use disorders identification test (AUDIT) in Korea. J. Kor. Acad. Fam. Med 20(9), 1152-1159 (1999).

37. First MB, Spitzer RL, Gibbson $M$, et al. Structured Clinical Interview for DSM- $\square$ Axis disorder. New York: New York State Psychiatric Institute (1996).

38. Jones JW. The children of alcoholics screening test: Test manual. Camelot Unlimited (1983).

39. Yum TH, Park YS, Oh KJ, et al. Manual of the Korean-Wechsler Adult Intelligence Scale. Seoul: Korean Guidance Press (1992).

40. Toplak ME, Sorge GB, Benoit A, et al. Decisionmaking and cognitive abilities: A review of associations between lowa Gambling Task performance, executive functions, and intelligence. Clin. Psychol. Rev 30(5), 562-581 (2010).

41. McKenzie M, Jorm AF, Romaniuk $H$, et al. Association of adolescent symptoms of depression and anxiety with alcohol use disorders in young adulthood: findings from the Victorian adolescent health cohort study. Med. J. Aust 195(3), S27 (2011).

42. Zung WW. A self-rating depression scale. Arch. Gen. Psychiatry 12(1), 63-70 (1965).

43. Spielberger CD, Gorauch RL, Lushene, RE. Manual for the state-trait anxiety inventory. Palo Alto, CA: Consulting Psychologists Press (1970).

44. Townshend JM, Duka T. Patterns of alcohol drinking in a population of young social drinkers: a comparison of questionnaire and diary measures. Alcohol. Alcohol 37(2), 187192 (2002).

45. Robinson OJ, Cools R, Carlisi CO, et al. Ventral striatum response during reward and punishment reversal learning in unmedicated major depressive disorder. Am. J. Psychiatry 169(2), 152-159 (2012).

46. Kodituwakku PW, May PA, Clericuzio $\mathrm{CL}$, et al. Emotion-related learning in individuals prenatally exposed to alcohol: an investigation of the relation between set shifting, extinction of responses, and behavior. Neuropsychologia 39(7), 699-708 (2001).

47. Brown EC, Hack SM, Gold JM, et al. Integrating frequency and magnitude information in decision-making in schizophrenia: An account of patients performance on the lowa gambling task. J. Psychiatr. Res 66-67, 16-23 (2015).

48. Bechara A, Damasio H, Tranel D, et al. Deciding advantageously before knowing the advantageous strategy. Science 275(5304), 1293-1295 (1997).

49. Bø R, Aker M, Billieux J, et al. Binge drinkers are fast, able to stop - but they fail to adjust. $J$. Int. Neuropsychol. Soc 22(1), 1-9 (2015).

50. Toplak ME, Jain U, Tannock R. Executive and motivational processes in adolescents with attention-deficit-hyperactivity disorder (ADHD). Behav. Brain Funct 27(1), 8 (2005).

51. Lin $\mathrm{CH}$, Chiu YC, Lee $\mathrm{PL}$, et al. Is deck $\mathrm{B}$ a disadvantageous deck in the lowa gambling task? Behav. Brain Funct 15(3), 16 (2007).

52. Johnson CA, Xiao L, Palmer $P$, et al. Affective decision-making deficits, linked to a dysfunctional ventromedial prefrontal cortex, revealed in $10^{\text {th }}$ grade Chinese adolescent binge drinkers. Neuropsychologia 46(2), 714726 (2008).

53. Gullo MJ, Jackson CJ, Dawe S. Impulsivity and reversal learning in hazardous alcohol use. Pers. Individ. Dif 48(2), 123-127 (2010).

54. Bø R, Billieux J, Landrø NI. Binge drinking is characterized by decisions favoring positive and discounting negative consequences. Addict. Res. Theory (2016).

55. Greening SG, Finger EC, Mitchell DGV. Parsing decision making processes in prefrontal cortex: response inhibition, overcoming 
Research Myung-Sun Kim

learned avoidance, and reversal learning. Neuroimage 54, 1432-1441 (2011).

56. Rushworth MF, Noonan MP, Boorman ED, et al. Frontal cortex and reward-guided learning and decision-making. Neuron 70(6), 1054-1069 (2011).

57. Holland PC, Gallagher M. Amygdala-frontal interactions and reward expectancy. Curr. Opin. Neurobiol 14(2), 148-155 (2004).

58. Schoenbaum G, Shaham Y. The role of orbitofrontal cortex in drug addiction: $A$ review of preclinical studies. Biol. Psychiatry
63(3), 256-262 (2008).

59. Bava S, Thayer R, Jacobus J, et al. Longitudinal characterization of white matter maturation during adolescence. Brain. Res 1327(1), 38-46 (2010).

60. Guerri C, Pascual M. Mechanisms involved in the neurotoxic, cognitive, and neurobehavioral effects of alcohol consumption during adolescence. Alcohol 44(1), 15-26 (2010).

61. Adan A, Navarro, JF, Forero DA. Personality profile of binge drinking in university students is modulated by sex. A study using the alternative five factor model. Drug. Alcohol. Depend 165, 120-125 (2016).

62. Scaife JC, Duka T. Behavioural measures of frontal lobe function in a population of young social drinkers with binge drinking pattern. Pharmacol. Biochem. Behav 93(3), 354-362 (2009).

63. Jones SE, Oeltmann J, Wilson TW, et al Binge drinking among undergraduate college students in the United States: implications for other substance use. J. Am. Coll. Health 50(1), 33-38 (2001). 